
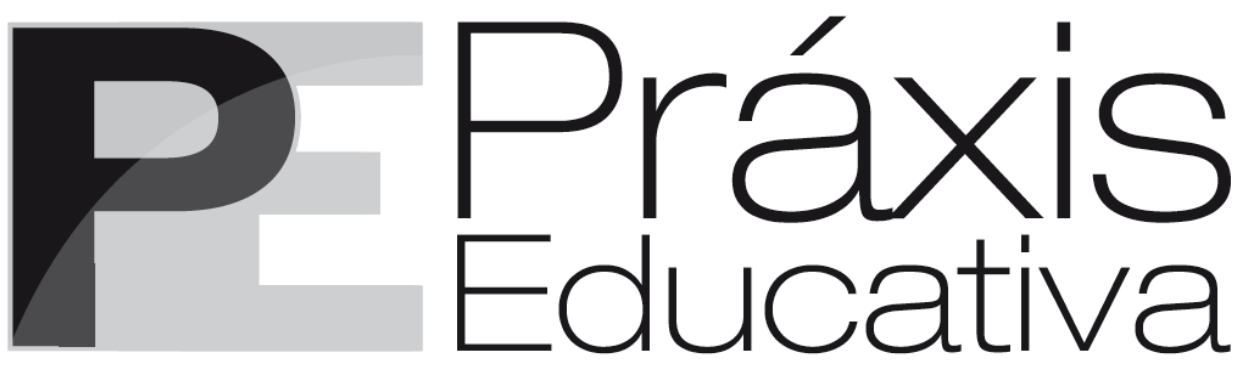

ISSN 1809-4309 (Versão online)

DOI: 10.5212/PraxEduc.v.12i3.002

\title{
"De intelectuales a expertos" en el campo educativo argentino de la década del sesenta. El caso del Centro de Investigaciones en Ciencias de la Educación ${ }^{*}$
}

\section{"From intellectual to expert" in the Argentinian education field in the 1960s. The case of the Center for Research in Educational Sciences}

\section{"De intelectuais a expertos" no campo educativo argentino da década de sessenta: o caso do Centro de Investigações em Ciências da Educação}

Jorgelina Mendez ${ }^{* *}$

Resumen: El presente trabaja indaga una institución particular, el Centro de Investigaciones en Ciencias de la Educación del Instituto Torcuato Di Tella, donde se combinan los elementos necesarios para definir un perfil de experto en educación. Durante la década de 1960 las ciencias sociales, incluyendo a las ciencias de la educación tuvieron un fuerte crecimiento y desarrollo que se ha denominado modernización. Ello generó nuevos perfiles académicos y profesionales entre los que se destaca el experto. En Argentina este proceso se enmarcó en un contexto de inestabilidad política y de conflictos entre los gobiernos de turno y las universidades. Tomando como referencia el contexto histórico se indagarán las características del centro, los investigadores que allí trabajaron y las actividades que se realizaron buscando caracterizar al experto en educación.

Palabras clave: Expertos. Modernización. Ciencias de la educación.

\begin{abstract}
This work investigates a particular institution, the Center for Research in Educational Sciences of the Torcuato Di Tella Institute, where the necessary elements were combined to define the profile of an expert in education. Throughout the 1960s the Social Sciences, including Educational Research, had a strong growth and development, which was called modernization. This generated new academic and professional profiles, where the expert stands out. In Argentina this process was generated in a context of political instability and conflicts between governments and universities. Taking the historical context into account, we analyzed the characteristics of the center, the researchers who worked there and the activities carried out during the first period of operation seeking to characterize the "expert" in education.
\end{abstract}

Keywords: Expert. Modernization. Education sciences.

\footnotetext{
* Este trabajo comunica las principales conclusiones del trabajo de investigación que enmarcó la tesis de Maestría en Ciencias Sociales y Humanidades (Universidad Nacional de Quilmes, Argentina) y que estuvo dirigido por la Dra. Ana María Montenegro y Co-dirigida por la Dra. Flavia Fiorucci.

${ }^{* *}$ Licenciada en Ciencias de la Educación y Magister en Ciencias Sociales y Humanidades con Mención en Historia. Becaria doctoral de CONICET. Miembro del Núcleo de Estudios Educacionales y Sociales (NEES) de la Universidad Nacional del Centro de la Provincia de Buenos Aires (UNCPBA). Profesora del área Historia de la Educación en la Facultad de Ciencias Humanas de la misma universidad. E-mail: <jorgelinamendez@gmail.com>.
} 
Resumo: Este trabalho investiga uma instituição particular, o Centro de Investigação em Ciências da Educação da Instituto Torcuato Di Tella, onde os elementos necessários se combinam para definir um perfil de "experto" em educação. Durante os anos 1960 as ciências sociais e também as ciências da educação tiveram um forte crescimento e desenvolvimento, chamado de modernização. Isto permitiu o desenvolvimento de novos perfis académicos e profissionais, onde destaca o "experto". Na Argentina este processo foi gerado em um contexto de instabilidade política e os conflitos entre sucessivos governos e universidades. Tendo em conta o contexto histórico, analisamos as características do centro, os pesquisadores que trabalhavam lá e as atividades que ocorreram durante os primeiros anos de operação procurando caracterizar a "experto" em educação.

Palavras-chave: Especialista. Modernização. Ciências da Educação.

\section{Introducción}

En la Argentina ha sido ampliamente estudiada la relación entre los intelectuales y la política y con ello, la inestable relación que las elites intelectuales mantuvieron con los gobiernos de turno, hecho que afectó principalmente a la institución universitaria que quedó sumergida en esos conflictos. A lo largo del siglo $\mathrm{XX}^{1}$ fueron comunes las intervenciones del poder político a las universidades, los despidos de docentes, la suspensión de actividades, la prohibición de la actividad política en sus claustros etc. Estos conflictos impulsaron a los intelectuales a generar espacios de producción y difusión del conocimiento independientes del poder del Estado y, por lo tanto, externos al ámbito universitario.

No obstante, el campo intelectual educativo por las características de su objeto de estudio, siempre ha debido estar estrechamente relacionado con el Estado y por lo tanto con los diversos gobiernos. Primeramente, por detentar hasta mediados del siglo pasado el monopolio de la oferta educativa oficial y, luego, por subvencionar gran parte de la oferta privada. En segundo lugar, porque es quien delinea la política educativa y gobierna la cúpula del sistema, aunque aquí caben otras discusiones que nos exceden. En síntesis, el Estado a través de los diversos gobiernos necesitó, obligadamente, referenciarse con miembros del campo intelectual y académico de la

\footnotetext{
${ }^{1}$ Hasta la década del '60 - punto de partida de este trabajo- es posible encontrar claros ejemplos de este tipo de conflicto. Tomando como punto de partida la Reforma Universitaria -impulsada en Córdoba en 1918 durante el gobierno del radical Hipolito Yrigoyen y que moldea a la universidad en términos "modernos"- podemos señalar los conflictos que se expresan con la interrupción del orden democrático en 1930. La restauración conservadora que siguió al golpe de estado no sólo suspendió los principios conquistados por el movimiento reformista, sino que, además, fue el espacio donde se evidenciaron los conflictos ideológico-políticos, al interior de la universidad y con los gobiernos nacionalistas y conservadores que se sucedieron. Estos conflictos se revitalizaron durante los gobiernos peronistas. Las universidades -como parte del ámbito intelectual- eran una importante fuente de resistencia a la figura del Presidente Perón tanto al interior del claustro docente como en las agrupaciones estudiantiles. Como contrapartida el peronismo sancionó una Ley universitaria - N$^{\circ} 13.031$ - que suprimía los principios de la Reforma Universitaria del '18: recortaba la autonomía universitaria, la elección de autoridades y docentes quedaba supeditada a aprobación del Poder Ejecutivo, prohibía la actividad político-partidaria en su interior y eliminaba la figura del co-gobierno restringiendo la participación de los alumnos a un representante con vos y sin voto en los órganos de gobierno siendo elegido por sus virtudes académicas (los mejor promedios de los últimos años). Esto provocó que un importante grupo de docentes fueran despedidos o decidieran renunciar a la universidad en función de su manifiesto antiperonismo. En un movimiento inverso, los grupos que promovieron el golpe de Estado al gobierno de Perón encontraron un aliado en la intelectualidad antiperonista, quienes tuvieron un rol importante en el proceso de "desperonización" de la sociedad y, también, de la universidad. Con un discurso de restauración del presunto orden democrático perdido (aunque se trataba de un gobierno instaurado por un golpe de estado y por lo tanto no democrático) la autodenominada "Revolución libertadora" reinstauró algunos principios de la Reforma del '18 (fundamentalmente el Co-gobierno) e inició un proceso tendiente a la normalización universitaria en el marco de la proscripción del peronismo. Este proceso incluyó el llamado a concurso de todas las cátedras con el impedimento de presentarse a tales concursos a docentes identificados abiertamente con el peronismo o que hayan accedido a las mismas durante ese gobierno.
}

Práxis Educativa, Ponta Grossa, v. 12, n. 3, p. 708-727, set./dez. 2017 Disponível em: <http://www.revistas2.uepg.br/index.php/praxiseducativa > 
De intelectuales a expertos" en el campo educativo argentino de la década del sesenta...

educación y estos intelectuales pudieron así participar de la elaboración de política como ministros, inspectores, reformadores, etc.

En la década del sesenta, en el marco de una trasformación del campo y de las relaciones con el poder de turno, se generaron las condiciones para que esa relación se complejice. Asimismo, en esos años se redefinió el perfil del intelectual en las ciencias de la educación ganando espacio la figura del experto, portador de otras características y de nuevas formas de intervención en el ámbito académico y en la esfera pública.

Este trabajo tiene como objetivo caracterizar desde la historia de la educación, la emergencia de la figura del experto en el campo educativo argentino indagando una institución singular que tuvo un peso fundamental en la delimitación de dicho perfil y en la institucionalización de la investigación educativa. Nos referimos al Centro de Investigaciones en Ciencias de la Educación (CICE) que funcionó como centro asociado del Instituto Torcuato Di Tella. Se trata de un análisis empírico y desde el marco de la historia social de la educación que recupera la mirada de una historia social de las ciencias sociales para el estudio de un caso específico y paradigmático.

El CICE se conformó en el marco del "Di Tella", en un momento de conflictividad política y social y de enfrentamiento entre gran parte del campo intelectual y la dictadura (19661973) en el gobierno. Es por eso que este centro, destinado a la investigación en educación, se configuró por fuera de la Universidad, en una institución privada, con una impronta diferente al modelo universitario local.

La trama de creación del CICE está marcada, además, por la de renovación que estaban viviendo las ciencias sociales en tanto campo académico y que impactó profundamente en las ciencias de la educación. La consolidación de nuevas teorías, disciplinas, objetos de estudios y metodologías, que pretendían un estudio científico del hecho social, se articuló con la demanda estatal, y desde los organismos internacionales, de información y datos que permitieran sustentar la toma de decisiones en el marco de elaboración de política pública. Mientras tanto, el perfil y la práctica del intelectual tradicional quedaban obsoletas bajo la demanda de un nuevo modelo profesional: el del "experto". Este trajo consigo nuevas formas de intervención y espacios de trabajo, en una especificidad disciplinar, en un delimitado campo de expertise.

El pasaje "del intelectual al experto" va a evidenciar otro tipo de relación con el estado, el gobierno y la política en general, en parte por los conflictos abiertos que la intelectualidad mantiene con el gobierno y, en parte, porque emergen otros espacios -por ejemplo, los organismos internacionales- desde donde legitimar ese conocimiento experto. Por lo tanto, dicho pasaje debe mirarse en relación con el contexto.

La indagación realizada muestra que al interior de este centro de investigación se gestaron las condiciones necesarias para que dicho proceso se iniciara en el marco de la redefinición del campo académico de las ciencias de la educación. Por lo tanto, esta comunicación pretende caracterizar al centro y su actividad mostrando las actividades, acciones y prácticas que permitieron la definición, primero, de un investigador en educación y luego, en ciertos casos, de un experto al que se denominará "experto/investigador".

El abordaje metodológico de la investigación tuvo un enfoque cualitativo y significó la triangulación de datos obtenidos de diversas fuentes analizadas. Asimismo, la sistematización de la información tuvo un aspecto cuantitativo que buscó tener referencias precisas sobre la cantidad de miembros, el número de investigaciones, las publicaciones, etc., con la intención de describir con datos certeros la actividad del centro y la importancia de la misma en el campo intelectual. 
Con respecto al corpus documental se relevaron por un lado, documentos del propio centro Memorias, balances, informes, documentos de trabajo y publicaciones- y, por otro, de diversos organismos con los que estaban relacionados ${ }^{2}$. Además, se realizaron entrevistas a ex miembros del instituto y se relevaron otras que guardaban relación con el objeto.

Para el desarrollo de nuestro objetivo en primer lugar se caracterizará al caso de estudio, el CICE, para luego desarrollar algunas discusiones teóricas sobre el análisis del campo intelectual de la educación. En segundo lugar, se presentarán las transformaciones del campo de la educación en relación al contexto histórico y las particularidades que permiten pensar que en este momento se gestan las condiciones para la definición de un "experto/investigador".

\section{El Centro de Investigaciones en Ciencias de la Educación como caso de estudio}

El CICE se fundó al interior del Instituto Torcuato Di Tella $a^{3}$ unos meses después de que la dictadura encabezada por el General Onganía (1966-1970) decretara la intervención de las universidades -en el mes de junio de 1966- y el despido de gran número de docentes acusados de "marxistas", como parte de la cruzada que ese gobierno llevó adelante contra el comunismo, y la izquierda en general de manera indiscriminada. En respuesta a esta acción, un gran número de docentes renunciaron a sus cargos, principalmente en las Facultades de Exactas y Filosofía y Letras de la Universidad de Buenos Aires (UBA), donde en esta última se dictaba la carrera Ciencias de la Educación.

Las renuncias y despidos se replicaron por el resto del país. Los docentes que, por la fuerza o voluntariamente, se fueron de la universidad representaban el crecimiento y modernización que esta había experimentado en la década anterior, más precisamente, desde su normalización en el año 1958. Algunos decidieron exiliarse y se incorporaron en universidades de distintas partes de América y Europa, otros emprendieron un "exilio interno" y buscaron la forma de continuar su tarea en otros espacios.

Este es el caso de los docentes que fundaron el CICE, entre los que se destacan su fundadora y directora, Gilda Lamarque de Romero Brest y los investigadores Ana María Babini, Hector Félix Bravo, Gregorio Weimberg, Celia Agudo de Córsico, Jaime Berstein, Enrique Oteiza, María Teresa Sirvent, Silvia Brusilovsky, entre muchos otros. En su totalidad se trataba de

\footnotetext{
2 Dichas instituciones son: el Instituto Internacional de Planeamiento, el Instituto Internacional de Educación, el Bureau internacional de Educación, todos ellos pertenecientes a Unesco, la Comisión Latinoamericana de Ciencias sociales (CLACSO), el International Development Research Center (IDCR) de Canadá, la Graduate School of Education y el Center for Studies on Education and Development de la Universidad de Harvard. Entre las nacionales se relevaron documentos pertenecientes a la, Fundación Bariloche, Consejo Nacional de Desarrollo (CONADE-Argentina), el Instituto Nacional para la enseñanza de las Ciencias (INEC- Ministerio de Educación). ${ }^{3}$ Dicho instituto fue promovido por los hermanos Guido y Torcuato Di Tella(h) hijos del empresario Torcuato Di Tella, industrial dueño de la fábrica Siam Di Tella. Guido y Torcuato (h) realizaron estudios de posgrado en importantes universidades de Estados Unidos (MIT y Columbia University) y trajeron de allí la visión de fundar en Argentina un instituto independiente para la producción de conocimiento. Su primer director, Enrique Oteiza, así como los investigadores principales de los primeros centros de investigación habían sido compañeros de estudios de los hermanos Di Tella. El instituto se organizaba en dos claras áreas el Centro de Arte -en la calle Florida en Buenos Aires- y los Centros de Ciencia -en diversos edificios en el barrio de Belgrano- estos tenían desarrollos independientes. Para profundizar sobre centros de arte y su rol en la vanguardia cultural consultar King (1985). Sobre la creación de los centros sociales se puede consultar Neiburg y Plotkin (2003).

${ }^{4} \mathrm{El}$ acontecimiento más destacado de este proceso de intervención y represión a las universidades acusadas de "reductos marxistas" a los que había que limpiar, fue la denominada "Noche de los Bastones Largos". Se trata del desalojo violento y la represión ejercida por las fuerzas de seguridad de las Facultades de Ciencias Exactas y Filosofía y Letras de la Universidad de Buenos Aires que estaban siendo pacíficamente ocupadas por docentes, estudiantes y autoridades como resistencia a la intervención y los despidos de docentes.
}

Práxis Educativa, Ponta Grossa, v. 12, n. 3, p. 708-727, set./dez. 2017 Disponível em: <http://www.revistas2.uepg.br/index.php/praxiseducativa > 
De intelectuales a expertos" en el campo educativo argentino de la década del sesenta...

docentes que habían renunciado a la universidad y buscaban de esta manera generar un espacio para continuar el trabajo iniciado al interior de la Carrera de Ciencias de la Educación de la UBA evitando la dispersión de las personas, pero también de sus producciones. En definitiva, tenían la intención de salvaguardar

10 años de esfuerzos orientados a formar un equipo cualificado y coherente de especialistas capaces de actualizar los estudios en Ciencias de la educación, renovar la carrera e impulsar la investigación. (ANEXO MEMORIA CICE, 1968, p. 43).

En las memorias se hace referencia a la "anexión" y no a la "creación" porque sus miembros lo reconocen como la continuación de la incipiente tarea de investigación que habían comenzado en el Instituto de Ciencias de la Educación en la Facultad de Filosofía y Letras de la UBA, aunque la misma no había alcanzado la sistematicidad y organicidad ni era una tarea expandida en el campo docente.

La conformación del CICE fue posible por un subsidio que su directora gestionó ante la Fundación Ford siendo el Instituto Di Tella quien le otorgó el marco legal e institucional para funcionar junto con el prestigio nacional e internacional con el que contaba. Su misión principal fue

Promover el estudio y la investigación en el campo de las ciencias de la educación especialmente en los problemas argentinos y latinoamericanos. Promover soluciones concretas a las cuestiones que el proceso de modernización plantea a la educación. (MEMORIA CICE, 1967, p. 3).

Este objetivo estaba a tono con las transformaciones que atravesaban al campo de la educación y que habían modificado su perfil disciplinar, así como de su campo profesional. Se organizaron equipos de investigación que llevaron adelante un importante corpus de estudios y trabajos sobre la base de las disciplinas que representaban sus miembros: la educación permanente, la política educativa, el planeamiento de la educación, la relación entre educación y desarrollo, la lingüística, el curriculum, etc.

Estas investigaciones estaban financiadas, en ciertos casos, por subsidios especiales a investigadores $\mathrm{y}$, en otros, por contratos con organismos nacionales e internacionales que demandaban estudios específicos. Al mismo tiempo, se desarrollaban trabajos de asesoría y consultoría para instituciones educativas, sindicatos, empresas y organismos estatales que requerían capacitaciones, estudios de oferta y demanda y se brindaban seminarios y cursos de perfeccionamiento para docentes.

Algunos de los miembros de mayor trayectoria -Gilda Romero Brest, Ana María Babini, Celia Agudo de Córsico, - fueron en esos años nombrados y contratados como expertos de UNESCO y del Consejo Latinoamericano de Ciencias Sociales (CLACSO), y lo hicieron en calidad de representantes de Argentina y del CICE, lo que marca la impronta y relevancia de este instituto.

Para todo el grupo de trabajo que componía el CICE este tipo de actividades suponía una novedad. En el interior de las universidades prevalecía la función docente y la organización estaba dada por la estructura de las cátedras y el trabajo que se hacía a su interior. Investigar, asesorar, participar de congresos, reuniones de especialistas, entre otras, eran actividades que fueron aprendiendo y desarrollando al tiempo que el centro se consolidaba.

De esta manera, se fue definiendo y acentuando el perfil de experto -lo cual suponía toda una novedad- al interior de una institución que tenía características muy diferentes a la universidad argentina pero que emulaba características de los centros de producción de 
conocimiento de Estados Unidos y de países europeos. El ITDT, como sus promotores la definen, era una institución privada, pero de "bien público" (ITDT, 1967), que se fundó con el objetivo de promover investigación de alto nivel para el desarrollo científico, cultural y artístico. Su principal intención era mantenerse ajena a los vaivenes políticos y económicos del país y evitar así la interferencia en la producción del centro. Para ello, creían que la mejor manera era ampararse en una Fundación, que llevaba el mismo nombre, y que estaba encargada de administrar los fondos propios del Instituto y aquellos que ingresaban en forma de contratos y donaciones desde una diversa cantidad de fuentes. Entre estas se destacan la Fundación Ford y la Fundación Rockefeller, la Agencia Internacional para el Desarrollo, el National Institute for Health odas ellas de origen norteamericano- y otras nacionales como Comisión Nacional de Administración de Fondos de Apoyo al Desarrollo Económico (CAFADE) -un organismo destinado a administrar el dinero distribuido en el marco de las acciones de la Alianza para el Progreso-, el Consejo Federal de Inversiones y la Organización de Estados Americanos. Algunos de estos organismos específicamente financiaron o ejecutaron contratos con el CICE para la producción de diferentes estudios e investigaciones.

Sin duda esto representaba un cambio en la forma de trabajo de quienes hasta ahora se definían mayormente como docentes universitarios. El campo intelectual educativo había estado, hasta el momento, relacionado con espacios públicos y estatales. Las universidades, Institutos de profesorado, escuelas normales, eran los ámbitos profesionales donde se desempeñaban y también cargos públicos al interior de la cartera educativa, como ministros, secretarios de educación, inspectores, entre otros. En el próximo apartado ahondaremos en algunas cuestiones teóricas que nos permitirán comprender mejor estas particularidades.

\section{Algunas precisiones teóricas y conceptuales}

En Argentina, la historia de las ideas y la historia intelectual han hecho foco en el campo de la literatura y la cultura en general al analizar el rol del intelectual y su relación con el Estado, es decir la configuración de una intelligentsia nacional. En esa caracterización, el intelectual educativo quedaba muy distante y poco reflejado ya que su rol era visible en relación con otra figura, la del funcionario público o el político "educativo".

La gran mayoría de los trabajos sobre las universidades y los grupos académicos e intelectuales en nuestro país se reconocen herederos de los aportes teóricos de Pierre Bourdieu. Los conceptos de campo, especialmente de campo intelectual y campo de poder, y en relación a estos últimos, el concepto de autonomización son lo que mayormente se utilizan para abordar la temática, aunque no sin cuestionamientos sobre las limitaciones que dichos conceptos presentan para analizar la complejidad de las relaciones entre campos, las influencias externas y la contextualización de los procesos (ALTAMIRANO, 2004; ALTAMIRANO; SARLO, 1993; SIGAL, 1991)

No obstante, en las últimas décadas comenzaron a publicarse una serie de trabajos que abordan la cuestión intelectual desde otro lugar. Su análisis se centra en emergencia de la cuestión social en Argentina (principios del siglo XX), en tanto problema de estado, analizando tanto al grupo intelectual preocupado por ella (ZIMMERMANN, 1995) como a la definición de un grupo de saberes que acompañaron la institucionalización de diversas disciplinas sociales (NEIBURG; PLOTKING, 2004; BLANCO, 2006). Estos últimos trabajos dan cuenta de la emergencia del experto dentro del campo intelectual, representante de un saber constituido, legitimado y puesto al servicio del Estado y portador de nuevas prácticas asociadas a esos saberes y, en paralelo, analizan también los espacios institucionales desde donde se desarrollaron, en algunos casos desde instituciones estatales y en otros por fuera de ella. De esta manera, el foco se vuelve sobre 
la expertise, es decir las características y posicionamientos que ocupa el experto en su práctica en clara relación con los espacios que ocupan profesionalmente y, por lo tanto, sobre la constitución de un campo de saberes profesionales asociados a él. Conjuntamente, otros trabajos estudian la conformación de profesiones y la institucionalización de esos cuerpos de saberes en relación con el aparato estatal (FREDERIC; GRACIANO; SOPRANO, 2010) así como también la configuración de elites intelectuales y burocracias ligada a dichos "saberes de estado" (ZIMMERMANN; PLOTKIN, 2012).

El común denominador de estos trabajos es, sin duda, el Estado como parte fundamental del proceso de institucionalización y definición de los campos disciplinares y profesionales. En el campo intelectual de la educación su rol tiene otro sentido adicional. No se trata, solamente, de la relación o el diálogo permanente con el Estado lo que colabora en la definición del campo, por el contrario, es el hecho que el propio campo emerge de la demanda de estos saberes y, quien demanda es principalmente el Estado.

Como plantea Suasnábar" (2013) es necesario atender a la noción "proceso de disciplinarización secundaria" de Hosftetter y Schneuwly (2002) para comprender la complejidad del campo educativo. Este concepto remite a la emergencia del campo disciplinar producto de un campo de saberes constituidos previamente en una práctica educativa institucionalizada y, por lo tanto, resultado de demandas externas que, en paralelo, es necesario adaptar a los cánones del conocimiento científico.

Tal como advierte Suasnábar, estas cuestiones pueden abordarse recuperando los aportes de un programa de investigación -relativamente reciente- que desarrolla una historia social de las ciencias sociales, dentro de las que se puede incluir a las ciencias de la educación. Es un esfuerzo por articular la "historia interna" de la disciplina y su propio desarrollo, con una "historia externa" que la atraviesa, la condiciona, la limita o la estimula (SUASNÁBAR, 2013). Conjuntamente se vuelve fundamental analizar las relaciones que la disciplina entabla con otras ciencias sociales y que forman parte de ese proceso de diferenciación externa que fija los límites del campo.

Hasta el momento, en la Argentina, el campo de "las ciencias de la educación" fue estudiado desde dos grandes aspectos. Por un lado, se encuentran trabajos abocados al análisis de los ámbitos institucionales (universidad-facultad-carreras) que van recortando el espacio académico de las ciencias de la educación y de un grupo de personalidades ligadas a ellas (CARLINO, 1993; SOUTHWELL, 2003a; SUASNÁBAR, 2004; OSSANNA, et al., 2010). En ellos se analizan los grupos académicos, el curriculum y las discusiones teóricas que sintetizan la producción y legitimización de un cuerpo de saberes y de personalidades autorizados a representar el campo Por otro lado, se ubican trabajos que analizan el campo desde la definición de grupos intelectuales, su inserción en el campo profesional y académico y la vinculación con la Universidad como espacio de generación de conocimientos o con el Estado como agente que demanda saber especializado para la toma de decisiones (SUASNABAR; PALAMIDESI, 2006; PALAMIDESSI; SUASNABAR; GALARZA, 2007; 2014; SUASNABAR, 2012; 2014).

El análisis que aquí se presenta busca complementar estos abordajes centrándose en la reconstrucción de una institución en particular, el CICE. Las actividades, relaciones, estrategias, en definitiva, las prácticas que allí se desarrollan permiten evidenciar cómo junto con la

\footnotetext{
5 Agradezco al Dr. Claudio Suasnábar, miembro del jurado que evaluó la tesis que generó los resultados que se comunican en este trabajo, los comentarios y sugerencias realizados durante la defensa de la misma y que contribuyeron en la realización de este artículo.
} 
modernización y actualización del campo académico, en este momento histórico emerge además una nueva figura: el experto.

La emergencia del experto, si bien tiene estrecha vinculación con la renovación del campo disciplinar, impacta en el campo intelectual al portar nuevas formas de trabajo y un cambio en el perfil profesional, en relación a la producción y circulación de conocimiento, que responde a las demandas del contexto, Las transformaciones del campo no sólo renovaron los temas a investigar y las metodologías a aplicar, sino fundamentalmente, transformaron el oficio del investigador lo cual supuso la adopción de nuevos roles, la incorporación de nuevas formas de trabajo y de validación del conocimiento científico, a tono con los nuevos marcos teóricos dominantes en ciencias sociales bajo el paradigma funcionalista.

La reconstrucción del trabajo realizado al interior del CICE permite evidenciar estas cuestiones. Se trata de un caso claro y preciso de modernización del campo y emergencia de nuevos roles y figuras que se definen a través de nuevas prácticas. Ello, junto con las características propias de la institución -al estar consolidada por fuera del ámbito universitarioposibilitará un tipo de trabajo intelectual y de difusión del conocimiento ya no ligado a la docencia y a la formación de docentes, sino a la representación de un saber experto.

\section{Re-definición del campo académico y disciplinar de las ciencias de la educación}

Hasta mediados del siglo XX el campo disciplinar de la educación en la Argentina estuvo dominado por un enfoque que ponderaba la dimensión filosófica de la educación y en donde la Pedagogía tenía el protagonismo absoluto. Si bien existieron experiencias interesadas en un análisis científico del "hecho educativo" - el trabajo de Victor Mercante y el laboratorio experimental de Paidología en la Universidad Nacional de la Plata, por ejemplo (DUSSEL, 1993) - con el decaimiento del positivismo como gran paradigma y, más específicamente en nuestro país, con el avance del espiritualismo en el campo educativo, la disciplina se unificó bajo el título Pedagogía y se dictó con ese nombre en las Universidades de Buenos Aires y La Plata hasta fin de la década de 1950. Por esos años, de la mano del paradigma funcionalista y en relación a los cambios sociales, políticos, culturales e internacionales del contexto, estas carreras se renovaron bajo un nuevo título: Ciencias de la Educación.

Para comprender dicho cambio hay que conocer el contexto en el que estaba inmersa la universidad. Entre 1958 y 1966 se gestaron las condiciones que permitieron la modernización de las universidades argentinas. Las transformaciones a nivel político, y las relaciones establecidas con el campo intelectual, facilitaron la inserción de nuevos discursos y prácticas que conllevaron la transformación de los planes de estudio, la creación de nuevas carreras en sintonía con las corrientes de pensamiento que dominaron el campo intelectual y la instauración de las actividades de investigación y extensión de manera sistemática, muchas de ellas financiadas a través de los organismos u agencias internacionales vinculadas a los problemas de desarrollo y otras desde los propios organismos creados desde el Estado para promover la investigación científica.

El proceso modernizador no estuvo ajeno a los vaivenes del sistema democrático de este período, tanto por las presiones externas como por las transformaciones internas.

Fue la UBA la que estuvo a la vanguardia de todas estas innovaciones y contó entre sus filas a los grupos académicos y científicos más destacados del momento y de la historia intelectual de nuestro país. Desde las universidades -y también desde el Estado - se llevaron adelante varias iniciativas que facilitaron la constitución de importantes grupos científicos en nuestro país. Una de ellas fue la política de exclusivización del claustro docente. Ello permitió que la labor 
De intelectuales a expertos” en el campo educativo argentino de la década del sesenta...

universitaria, reconocida por su prestigio social pero no por la posibilidad de vivir de ella, fuera una opción de desenvolvimiento profesional posible para los egresados de las carreras no profesionalistas. Esta política tuvo un fuerte impacto, Sigal (1991), advierte que en el conjunto de la UBA había un total de 2 docentes con dedicación exclusiva en el año 1955 y para 1962 eran 200 profesores y 524 asistentes exclusivos concentrados mayormente en las facultades de Ciencias Exactas y Filosofía y Letras. Es importante señalar que estas dos facultades lideraron el proceso modernizador y, en muchos casos, fueron las encargadas de librar las batallas con los resabios del viejo modelo.

La formación de científicos se vio facilitada por una política científica de Estado que se concreta con la creación de la Comisión de Investigaciones Científicas y Técnicas (CONICET) en 1958, teniendo como misión "promover, coordinar, y ejecutar investigaciones, en el campo de las ciencias puras y aplicadas, dentro de su campo de competencia" (LEY DE CREACIÓN DEL CONSEJO NACIONAL DE INVESTIGACIONES CIENTÍFICAS Y TÉCNICAS $\mathrm{N}^{\circ}$ 1.291/58). Para ello se creó un sistema de Becas para la realización de estudios de posgrado y la creación de la carrera de Investigador científico y tecnológico ${ }^{6}$.

Asimismo, organismos gubernamentales como el Consejo Nacional de Desarrollo (CONADE) demandaban egresados de las nuevas carreras como Sociología, Economía y Ciencias de la Educación ${ }^{7}$ para incorporar a sus filas como especialistas o expertos de las diversas áreas con el fin de generar las bases para las políticas de desarrollo que demandaba el país ${ }^{8}$. Esta se convirtió en otra vía de desarrollo profesional acorde con la formación académica que brindaba el nuevo plan de estudios de "Ciencias de la Educación", en sintonía con la nueva denominación de la carrera.

La modernización de las ciencias sociales fue una preocupación de las autoridades de la UBA desde su normalización, motorizada bajo las rectorías de José Luis Romero y Risieri Frondizi. Dicha modernización incluyó la creación de carreras (Sociología, Psicología, Ciencias de la Educación y Ciencias Antropológicas), la renovación de planes de estudio, la departamentalización de la facultad de Filosofía y Letras, y la renovación de su planta docente

Las nuevas carreras facilitaron la transformación de la estructura de la facultad de Filosofía y Letras marcando un quiebre respecto de los "estudios basados en la cultura clásica" (BUCHBINDER, 1997, p. 195) que representaban a carreras como Historia y Filosofía. Las

\footnotetext{
${ }^{6}$ En función de los números expuestos por Sigal (1991) en base a la fuente Informe de CONICET la evolución en el caso de las becas fue de 54 para el primer año y de 124 para 1966 en el caso de las internas y en el caso de las externas el número inicial de '63 se mantuvo estable con picos de 93 y 87 en los años '61, '62 y '63. En el caso de los puestos de investigador, los mismos variaron de 214 en el año '61 a 297 en el '66 incrementándose año a año. (Nota 8 p. 87)

${ }^{7}$ Southwell (2003b) destaca entre ellos a Roberto Fernandez Lamarra (quien estuvo a su cargo), Inés Aguerrondo, Sara Morguesten de Finkel, Lucila Arigazi, Silvia Llomovate, Alicia Vales, Norma Paviglianiti, Hebe Lopez, María Teresa Farrés, David Wiñar, Susy Finkel, Graciela Riccó, y economistas como Guillermo Fischman” (p. 123), todos ellos se desempeñaron en el "Sector Educación" del CONADE creado en 1964.

${ }^{8}$ EL CONADE fue creado en 1961 durante la presidencia de Arturo Frondizi. Se trató de un organismo con una marcada impronta Desarrollista, que surgió en el marco de los compromisos asumidos de la Alianza para el Progreso (ALPRO) y bajos lineamientos directos de CEPAL. El principal objetivo de este organismo era orientar el desarrollo en todos sus aspectos pero, fundamentalmente, desde la planificación económica teniendo en cuenta que los organismos de crédito ya comenzaban a solicitar que los pedidos de financiamiento se encuentren encuadrados en planes o proyectos integrales. Entre los proyectos más ambiciosos de este organismo podemos mencionar el "Plan Nacional de Desarrollo 1965-1969" -que estuvo en vigencia durante la presidencia de Arturo Illia- y, a nivel educativo, el trabajo publicado bajo el nombre "Educación, recursos humanos y desarrollo" en el año 1968 durante el gobierno de facto del Gral. Onganía.
}

Práxis Educativa, Ponta Grossa, v. 12, n. 3, p. 708-727, set./dez. 2017 Disponível em: <http://www.revistas2.uepg.br/index.php/praxiseducativa> 
nuevas disciplinas privilegiaron el trabajo empírico, las observaciones y el trabajo de campo frente a una fuerte tradición antipositivista (op.cit).

Precisamente este fue el caso de la carrera de Pedagogía, la antecesora de las Ciencias de la Educación, con fuerte raíces en la Filosofía y de corte espiritualista. Como superación de este enfoque, según señalan Sirvent y Llomovate (2007), la carrera Ciencias de la Educación se orientó hacia un estudio científico del hecho educativo en el marco de las ciencias sociales. El nuevo plan de estudios marcó lo que Carlino (1993), retomando a Carlos Altamirano y Beatriz Sarlo, denomina una nueva problemática dominante del campo orientada a una formación científica y profesional

\begin{abstract}
Por un lado, el método científico y la investigación de base empírica como instrumento por excelencia de producción de conocimiento, tienen una abrumadora presencia en el contenido temático de los programas de distintas materias (...) Las palabras claves que aparecen con recurrencia son: 'lógica deductiva', 'hipótesis', 'explicación', 'teoría'. Por otro lado, la teoría social funcionalista se palpa con los autores 'faro' leídos y difundidos a través de la carrera (G.GERMANI, MALINOWKY, PARSONS) y en la presentación temática del contenido de algunas materias. (CARLINO, 1993, p. 52) (Las mayúsculas corresponden al original).
\end{abstract}

Es posible observar en esta descripción cómo las ciencias de la educación se pusieron a tono con las discusiones que dieron sustento a las ciencias sociales en general. Sin embargo, no sólo el funcionalismo formó parte de esta nueva construcción teórica. También el marxismo fue ganando lugar e "ingresó" al espacio universitario desde las nuevas generaciones

Carlino (1993) observa que en el primer plan de estudios del año 1958 sobreviven seis materias, algunas del tronco común y otras optativas, que representan el campo filosófico del anterior plan de Pedagogía ${ }^{9}$. Como advierte Carlino y refuerza Suasnábar (2004) en este nuevo plan de estudios se pueden observar las tensiones entre las problemáticas dominantes de las ciencias sociales y aquellas que perduran del paradigma anterior. Estas tensiones expresan que la modernización de la disciplina no fue un proceso lineal ni total al interior de la UBA, que encontró resistencias y no se modeló completamente durante estos años. Esta situación se observa también en otras universidades como La Plata (SOUTHWELL, 2003a), la Facultad de Ciencias de la Educación de Paraná (KUMMER, et al., 2010) y el Instituto Universitario de Tandil (MÉNDEZ, 2014).

Si retomamos el caso de la UBA, el diálogo con las otras disciplinas sociales que surgieron en la misma facultad fue lo que facilitó el ingreso de nuevas perspectivas teóricas, tal como advierten Carlino (1993) y Southwell (2003b). Las profesoras Ana María Babini y Gilda Lamarque de Romero Brest -quienes posteriormente tendrán un lugar preponderante al interior del CICEfueron dos exponentes de esta renovación. La primera formó parte del grupo de investigadores ligados a Gino Germani fundador de la "sociología científica" en nuestro país, y fue pionera en el ámbito de la Sociología de la Educación. La segunda, aunque discípula de Juan Mantovani exponente del espiritualismo pedagógico- en la cátedra de Pedagogía, fue una de las precursoras del funcionalismo pedagógico en nuestro país e impulsora de la transformación del campo

Gilda [Lamarque de Romero Brest] personificaba en aquel momento, para los jóvenes de la carrera, la innovación en ciencias sociales y en Educación. Esto le valió

\footnotetext{
9 Carlino (1993) señala las siguientes: "Introducción a la Filosofía" y "Ciencia y Filosofía de la Educación" obligatorias de primer y cuarto año respectivamente. "Filosofía de las Ciencias", "Historia de la Filosofía (antigua y medieval)", "Historia de la Filosofía (moderna y contemporánea)" y "Filosofía de la Historia" optativas de segundo a cuarto año.
} 
De intelectuales a expertos” en el campo educativo argentino de la década del sesenta...

profundos conflictos y confrontaciones con los profesores de la carrera que provenian de la antigua carrera de Pedagogía y que cuestionaban los enfoques que ella introducia, como ser la visión de un estudio cientifico del fenómeno educativo. (ENTREVISTA $\left.1^{10}\right)$.

En el año 1961, luego del fallecimiento de Mantovani, la profesora Gilda L. de Romero Brest, asumió la dirección del departamento de educación en la Facultad de Filosofía y Letras. Este sufrió un proceso de transformación para adaptarse a las demandas del desarrollo y así llevar adelante un programa de modernización

que incluía la solicitud de becas para el perfeccionamiento de graduados en el extranjero, el acrecentamiento del personal docente, el dictado de cursos de formación de auxiliares docentes universitarios y la producción de materiales didácticos de circulación interna. (CARLINO, 1993, p. 50)

Estas iniciativas formaban parte de la transformación de la carrera de Pedagogía en la de Ciencias de la Educación, es decir, la asunción del estudio científico del hecho educativo a tono con las necesidades del contexto y en el marco de un proyecto de ciencia social ampliada el que se entrelazan diversas tradiciones y posiciones epistemológicas que entran en disputa.

Estas disputas ejercieron presión sobre la modernización del campo en lo disciplinar, pero, más allá de la renovación de los planes de estudio, no generó instancias claras de investigación al interior de las carreras, aunque el "terreno ya estaba fértil". Todas estas transformaciones contribuyeron a la constitución de una masa crítica de docentes, potenciales investigadores, con la capacidad para llevar adelante proyectos de diversa índole.

Sin duda el proceso que se abrió al interior del CICE no hubiese sido posible sólo por la renovación de ese campo específico. Fundamentalmente, se pudo llevar adelante, por la puesta en marcha de todo un proceso de renovación que envolvió a la Universidad y que se vivió más claramente en la UBA. La interrupción de este proceso luego de la intervención perpetrada por el gobierno de facto del General Onganía, impulsó a las ciencias de la educación para que ese potencial continuara desarrollándose fuera de los muros universitarios. Es por lo tanto que afirmamos que el CICE se constituyó en continuidad con ese proceso afianzado en la universidad promoviendo aquello que quedó inconcluso de su proyecto: la institucionalización de un campo de investigación.

\section{Institucionalización de la investigación: el trabajo académico al interior del CICE}

El análisis de las memorias del centro de investigación, las entrevistas relevadas así como aportes de otras investigaciones permiten afirmar que el CICE se reveló como un objeto informador clave para reconstruir los cambios de las ciencias de la educación en el marco general de las ciencias sociales y, fundamentalmente, para identificar un nuevo perfil en el oficio del investigador que, al tiempo que se aggiorna a las exigencias de los nuevos paradigmas científicos, mantiene elementos de continuidad con etapas previas.

\footnotetext{
${ }^{10}$ Entrevistada 1. Mujer, graduada de la carrera Profesorado de en Ciencias de la Educación. Fue jefa de trabajos prácticos de la asignatura Pedagogía de dicha carrera en la UBA. Formó parte del plantel del CICE como Investigador asistente hasta el año 1969 cuando se trasladó a los Estados Unidos a cursar sus estudios de posgrado. La entrevista fue tomada en mayo del 2009 por investigadores de la Universidad de Buenos Aires para un proyecto que recopila la Historia Oral de la carrera de Ciencias de la Educación y cedida por la entrevistada quien coordinaba dicho proyecto. Desconocemos los nombres de los entrevistadores quienes tienen el crédito por la misma. Fue editada y corregida por la entrevistada.
}

Práxis Educativa, Ponta Grossa, v. 12, n. 3, p. 708-727, set./dez. 2017 Disponível em: <http://www.revistas2.uepg.br/index.php/praxiseducativa $>$ 
El CICE emergió, en el centro de los cambios sociopolíticos de la década del '60, como una institución extra-universitaria que canalizo un impulso modernizador que ya se venía desarrollando al interior de la carrera de ciencias de la educación de la UBA, en cuanto a lo disciplinar y que allí se materializó en el oficio del investigador.

Es significativo que el campo educativo en general señala la recuperación democrática de 1983 como el punto de partida para la institucionalización de la investigación educativa tanto al interior como al exterior de las universidades (GALARZA, 2007; TIRAMONTI, 2004; 2016). Sin duda la sistematicidad, organicidad y progresividad de las investigaciones educativas no fue posible hasta que la estabilidad democrática permitió sustentar estos proyectos, garantizando la libertad para llevarlos adelante, aunque no siempre acompañados desde el financiamiento. No obstante, el análisis de este caso de estudio muestra que las condiciones ya estaban generadas desde la renovación del campo disciplinar y que fue al interior del CICE donde se dieron los primeros pasos y se gestaron los primeros recursos humanos que, posteriormente, harán florecer al campo.

Es necesario aclarar que el CICE no fue el único espacio desde donde se desarrollaron actividades de investigación además de los organismos estatales de planificación como el Sector Educación del CONADE. La relación que tendrán los diversos espacios que se configuran en estos años con la posterior institucionalización de la investigación educativa es sumamente significativa. Los primeros graduados de Ciencias de la Educación, así como los jóvenes ayudantes de cátedra que contribuyeron a su modernización, fueron quienes, con la vuelta de la democracia, motorizaron la investigación al interior de las Universidades -por ejemplo refundando el Instituto de Ciencias de la Educación de la UBA- , al interior del Estado -desde diversos organismos que los contrataron para realizar diagnósticos, evaluaciones, relevamientos de situación, etc.- y en nuevos espacios privados como el sector educación de la Facultad Latinoamericana de Ciencias Sociales creado en 1982.

Algunas de las figuras que se destacan en estos espacios en la vuelta de la democracia -tal es el caso de María Teresa Sirvent, Silvia Brusilovsky, Norma Paviglianiti, David Wiñar, Graciela Riquelme y la propia Gilda L. de Romero Brest, tuvieron, en mayor o menor medida, un paso por el CICE. Este fue el ámbito donde generaron habilidades y prácticas que permitieron que consolidaran un perfil de investigadores.

Precisamente una de las entrevistas ${ }^{11}$ sugiere que el CICE fue un espacio de refugio y contención -ante la intervención universitaria- para todo un grupo de profesores que venían trabajando por la modernización de la carrera de Ciencias de la Educación pero que tenían muy poca trayectoria como investigadores. Ella plantea que al momento de la intervención militar y ante el despido y la renuncia a la universidad "los equipos de cátedra se rearmaron como equipos de investigación" y que en distinta medida fueron desarrollando tareas de investigación:

Los otros equipos estaban constituidos también por renunciantes, las caberas de los equipos, eran los titulares, los que babian sido profesores titulares.

En la cátedra no haciamos investigación. Habiamos empezado, yo no, habiamos empezado un poquitito cuando estaba en extensión universitaria pero muy poquito $y$ no avañó. Nos formábamos cada gradualmente cada uno en su equipo. Es decir, no babia un proceso de formación separado de la práctica misma. Nos íbamos formando

11 Entrevista 2. Mujer, graduada de las primeras promociones de la Carrera de Profesorado en Ciencias de la Educación. Ayudante de cátedra de las asignaturas Pedagogía e Introducción a las Ciencias de la Educación al momento de la intervención universitaria. Miembro del CICE entre 1966 y 1979 primero como asistente de investigación y luego con categoría de Investigador por concurso. La entrevista fue tomada en julio del 2012.

Práxis Educativa, Ponta Grossa, v. 12, n. 3, p. 708-727, set./dez. 2017 Disponível em: <http://www.revistas2.uepg.br/index.php/praxiseducativa> 
De intelectuales a expertos" en el campo educativo argentino de la década del sesenta...

como podíamos y a los ponchazos a medida que iban apareciendo los trabajos. Yo no sé cómo funcionaban los otros equipos, nosotros discutíamos entre nosotros, participábamos, preparábamos los diseños, eh, discutíamos la elaboración de los instrumentos, a veces aplicábamos nosotros los instrumentos otras veces contratábamos, era la época de la técnica de la encuesta, contratábamos encuestadores muchas veces. Pero nosotros procesábamos la información, bueno, aprendíamos en el proceso de investigación mirá, como diría Bourdieu, a investigar se aprende investigando. (ENTREVISTADA 2).

En otra entrevista se observan diferencias con el testimonio anterior al plantear un mayor grado de sistematicidad de las actividades allí organizadas, así como la existencia de equipos de investigación al interior de la UBA. No obstante, en ambos casos, se resalta el valor que tuvo para la formación de una nueva generación que asumió como una tarea fundamental, para el campo disciplinar, el producir conocimiento:

El espiritu fundador [del CICE] era generar un continente de continuidad en Investigación en Ciencias de la Educación frente al vaciamiento de la UBA y la inexistencia de otros espacios que la reemplazaran. A través del CICE, se organizaron talleres de investigación, y seminarios de formación y capacitación docente en Capital Federal y en provincias del interior del pais. La experiencia de esos seminarios, sobre todo en las provincias constituyó para mi una fuente inagotable de conocimiento. Desde mi perspectiva, el CICE fue, no sólo para los que habiamos sido docentes de la UBA sino para mucha gente más joven que se acercó a la institución un espacio importante de formación en ese momento de la historia del país y de la UBA. (ENTREVISTADA 1)

Con el fin de contrastar esta información se recopiló toda la información disponible en las memorias relevadas -entre el año 1966 y 1974- sobre las investigaciones realizadas y los equipos de investigación y realizamos un seguimiento a lo largo de los años triangulando lo allí dispuesto con otras fuentes de información. Se analizaron las nóminas de personal, las investigaciones, publicaciones, la información sobre los estudios de posgrado de los miembros y las actividades realizadas en vinculación con dichos centros. Al mismo tiempo, se relevó, en los casos que fueron posibles, las producciones finales de dichas investigaciones muchas veces resguardadas en los organismos con los cuales se vincularon.

Dicho relevamiento permitió la sistematización de la información disponible y el análisis de todas las investigaciones mencionadas en las memorias del CICE y del ITDT entre los años 1967 y 1973, el financiamiento con que contó, los integrantes del equipo y su continuidad en el tiempo, lo cual permitió observar algunos puntos clave. El corpus de datos no es homogéneo en todos los casos, con lo cual algunos se complementaron de otras fuentes. Mientras que en algunas podemos ver toda su evolución, otras dejan de constar en las memorias sin que se indique si finalizó, fue cancelada o suspendida.

Del análisis de la información podemos ver cómo algunos integrantes se repiten alrededor de un investigador o temática y, al mismo tiempo, otros que trabajan en solitario, o por lo menos eso se infiere de los datos de las memorias. Aunque tenemos indicios de que no en todos los casos los investigadores mencionan a su equipo en dichas memorias.

Asimismo, pudimos ver la rotación y estabilidad de los grupos. Mientras que algunos se mantienen ligados a una temática general, otros surgen vinculados a una investigación puntual e incluso mantienen una misma investigación a lo largo de todo el período.

Práxis Educativa, Ponta Grossa, v. 12, n. 3, p. 708-727, set./dez. 2017 Disponível em: <http://www.revistas2.uepg.br/index.php/praxiseducativa > 
El número de investigaciones activas en cada año se mantiene más o menos estable con un total de 12 en el año ' 67,15 para los años ' 68 y '71 y 16 para el ' 73 . Un pequeño número de investigaciones se mantienen a lo largo del período '67-73 aunque algunas cambian sus integrantes o van dando cuenta de diferentes etapas de trabajo. En algunos casos podemos advertir que su finalización coincide con la publicación (libro, artículo, Documento de Trabajo, Documento Interno) pero no en la totalidad de los casos en que se presenta como finalizada.

Otra de las cuestiones relevantes que se evidencian en este análisis es el universo de instituciones que financian investigaciones radicadas en el CICE, representadas en su mayoría por UNESCO en sus diferentes institutos (Instituto Internacional de Planeamiento, IIPE, París y el Institute for Education -actual Institute for Lifelong Learning-, Hamburgo). Ello sumado a los vínculos establecidos con UNESCO por la directora del CICE permite afirmar que se trataba de un organismo vital para el funcionamiento de este centro. Las temáticas de esas investigaciones en particular se inscriben en los temas en boga del período -rendimiento escolar; planeamiento de la educación; educación permanente- temas a los que los institutos de UNESCO dedicaron seminarios internacionales, publicaciones y capacitaciones. Además de las temáticas mencionadas se destacan, la relación educación - desarrollo, educación y pobreza, política y financiamiento de la educación y, en menor medida, lingüística, curriculum y enseñanza, educación técnica, entre otros.

En síntesis, se observa una importante preponderancia de trabajos demandados o financiados por los organismos que se mencionaron. Este tipo de trabajo facilitó o posibilitó, la construcción de un campo de experticia que delimitará una nueva tarea -la investigación- y junto con ella la definición de una nueva figura -el experto. Ambas suponen una novedad para el campo de la educación y abren el camino a una nueva práctica profesional.

\section{De intelectuales a expertos: la modernización y profesionalización del campo}

A lo largo del siglo XX, asistimos a la "profesionalización" (GONZALEZ LEANDRI, 1999) de los campos de conocimiento y también al pasaje de un intelectual de tipo "generalista" a otro de tipo experto. Este último está dotado de un dominio técnico sobre un determinado campo de saber, capaz de intervenir en la solución de problemas concretos y en la elaboración de políticas para tal fin (CAMOU, 1997). La característica central del experto es el entrenamiento prolongado y la selección por méritos (GONZÁLEZ LEANDRI, op.cit) con lo cual cada vez más el reconocimiento y legitimidad del Experto provienen de la especialización de su formación y conocimiento y la inserción en ámbitos de investigación sistemática -universitarios o extra universitarios- con la consecuente participación en actividades científicas de carácter periódico como congresos, simposios o publicaciones. Por lo tanto, al definir al experto que emerge en este contexto se lo reconoce como un "experto-investigador", lo cual supone una novedad para el campo de las ciencias de la educación a fines de la década de 1960.

A principios del siglo $\mathrm{XX}$, el intelectual del campo educativo tenía características diferentes, fundamentalmente, porque no se trataba de un intelectual "puro", detentaba también un marcado rol político (MENDEZ, 2011). Es posible observar que su trascendencia en el campo intelectual estaba acompañada por su participación en el gobierno educativo desde diferentes cargos: ministros, inspectores, rectores y directores de universidades e institutos, etc. Por lo tanto, se trataba de un "intelectual/funcionario", es decir aquel que combina su trayectoria académica, su aporte a la construcción de conocimiento del campo educativo, con su participación en diversos espacios y niveles de gestión y conducción del sistema educativo. 
De intelectuales a expertos” en el campo educativo argentino de la década del sesenta...

El avance de la profesionalización y la consolidación de un conocimiento experto de dio en paralelo con la definición de una función técnica ligada a él. Se trata de dos caras diferentes del proceso. La figura del técnico portador de un saber legitimado, especializado, que emerge en la segunda mitad del siglo XX, se define por las nuevas demandas estatales que reclaman información -mayormente en forma de datos- para la toma de decisiones. Podríamos decir que se trata del rol "funcionario" de aquel intelectual educativo que definimos previamente. También requiere de un conocimiento experto - o especializado -, pero no significa que el experto sólo ocupe cargos técnicos. Por el contrario tiene un rol central en la producción de conocimiento bajo los nuevos parámetros de la investigación social- y por eso lo conceptualizamos "expertoinvestigador".

Estos cambios en los perfiles, roles y prácticas que se definen al interior del campo muestran la existencia de un pasaje entre el perfil intelectual y el perfil experto, pero no en términos de un remplazo. Por el contrario, da cuenta de una brecha abierta, de un espacio de frontera, que los comunica y que marca un desplazamiento en ambos sentidos y que se define en la complejidad del campo educativo.

Sin dudas gran parte de este pasaje se generó al interior del CICE. No fue azar, por el contrario, allí se condensó - en un clima propicio- el trabajo con los vínculos disciplinares e institucionales pertinentes para que emerja la figura del experto en educación. Dicha afirmación se sustenta en la importancia que adquirió el hecho de que este grupo de docentes se haya organizado como "centro de investigación" y, más específicamente, lo hayan hecho al interior del ITDT permitiendo desarrollar el perfil del investigador que no se había desenvuelto completamente en el marco universitario, a diferencia de otras disciplinas sociales.

Rearmar los equipos de cátedra como equipos de investigación fue un desafío para el centro y sus integrantes, que hasta el momento sólo habían trabajado bajo el marco que ofrecía la universidad pública, con la docencia como tarea primigenia, y que aún, en su época de oro, no había producido los suficientes antecedentes para encaminar la tarea específica de un centro de investigación. Es por ello que, para la configuración del CICE, es importante el marco que ofrece el ITDT el cual le permite extrapolar formas de organización, tareas y vínculos con otras organizaciones para su propio desarrollo.

De esta manera, el perfil que se va desarrollando dentro del CICE es el de investigador que, al mismo tiempo que se configura, va gestando las condiciones para la formación de nuevas generaciones portadoras de esa impronta y para lo cual se organiza una estructura -con mayor o menor nivel de explicitación- de formación de recursos humanos.

Se delimitan categorías de investigadores -en jefe, principal, asistente, entre otras. Los más jóvenes, no sólo participaban en las investigaciones, además se fueron incorporando en algunas publicaciones y varios de ellos tuvieron la posibilidad de cursar estudios de posgrado en el exterior y alcanzar el grado de Doctorado a través de las distintas becas ${ }^{12}$. Las entrevistas analizadas evidencian que la prosecución de estudios de posgrado era un proceso individual y no una acción sistematizada y organizada desde el interior del CICE pero que, en todo caso, había un acompañamiento por parte de su directora -Gilda L. de Romero Brest- y los investigadores Senior, que a través de contactos facilitaba estas gestiones y la obtención de recursos.

\footnotetext{
${ }^{12}$ Los casos más destacados y de los que se encontró información certera sobre su finalización son los de María Teresa Sirvent en la Universidad de Columbia, EE.UU., Abraham Paín en la Ecole pratique de Houtes Etudes, Francia, Susana Morgensten en la Universidad de Essex, Inglaterra.
} 
Como ya se afirmó, aquí se evidencia nuevamente el rol central de los diversos organismos con los que se vinculó el centro ya que, con el sustento o financiamiento directo de estos organismos, se realizaron investigaciones, actividades académicas, formación de recursos humanos, etc. Así, el perfil del investigador adquirió un plus. Se volvió un personaje a ser consultado o contratado para diseñar y llevar adelante proyectos, opinar sobre cuestiones relativas a la temática educativa general, pero fundamentalmente, sobre aquellas en las que está especializado.

En las investigaciones realizadas desde el CICE, es posible observar que aquellas financiadas por organismos internacionales o gubernamentales son, fundamentalmente, diagnósticos de situación, elaboración de modelos conceptuales, estudios exploratorios, es decir, trabajos que apuntan al análisis de la realidad y a la intervención en ella desde diferentes aproximaciones. Pero, sin duda, donde más claramente se muestran las acciones que caracterizan a la figura del experto es en las actividades llamadas "consultorías".

Hay diversos tipos de actividades comprendidas en dicha categoría, no obstante, aquellas más significativas, son las que se organizan desde organismos internacionales y culminan con "seminarios" o en "reuniones de expertos". En estas últimas, como el propio título indica, los miembros del CICE eran invitados a participar en reuniones donde se requerían sus opiniones o contribuciones sobre alguna temática en particular. Este tipo de reuniones, seminarios o conferencias internacionales se realizaban -y se realizan- sobre una gran variedad de temas y problemáticas del campo educativo. La indagación sobre las memorias mostró que, año a año, algunos investigadores del CICE participaban de estas actividades en las cuales eran considerados "expertos"13.

Nuevamente, se puede ver a los miembros la intervención en calidad de autoridad en la materia, de especialista. Ese plus que mencionamos anteriormente, aquello que excede la práctica de lo que llamamos un "investigador" y que lo vincula con organismos internacionales, gubernamentales y no gubernamentales, en investigaciones, asesorías, consultorías, etc., es lo que constituye, en nuestro caso, la figura del experto en educación.

Así como definimos al intelectual en educación de principios del siglo XX lo definimos como un intelectual/funcionario, en este contexto, cuando hablamos del experto, percibimos un movimiento que se mueve entre el investigador/experto y el experto/investigador. En tanto investigador/experto legitima, ante los organismos -estatales e internacionales- que lo convocan, su autoridad y la del centro al que representa. En tanto experto/investigador trae de los organismos por los cuales ha sido convocado, un valor, un prestigio, que lo posiciona a él y al centro al que pertenece. Es por ello que, en parte, describir el perfil del experto es también describir la delimitación del perfil del "investigador formado". Decimos en parte porque también es describir ese plus que señalamos.

\footnotetext{
${ }^{13}$ Entre las acciones que constan en las memorias del centro se destaca la participación de Ana María Babini en el Seminario "Social Background of students", International Bureau d' education, UNESCO, Ginebra y Comisión de trabajo "Educación y desarrollo", CLACSO, México y Lamarque de Romero Brest en la Reunión de expertos "La contribución de la antropología educativa y de la sociolingüística al desarrollo de la educación” UNESCO, París; la Reunión de expertos acerca de "El impacto de la educación permanente. en la innovación escolar y de Curriculum" UNESCO, Instituto de Educación, Hamburgo y el Consejo de gobernadores del Institute for education, UNESCO, Hamburgo.
} 
De intelectuales a expertos" en el campo educativo argentino de la década del sesenta...

\section{Conclusiones}

Este recorrido puso en evidencia que la redefinición y modernización disciplinar y profesional de las ciencias de la educación fue producto de múltiples condicionamientos y demandas. Conjuntamente se demostró que la institucionalización del campo disciplinar y la definición de un perfil de "experticia", lo que hemos llamado el experto/investigador, fueron dos procesos fragmentados en tiempo y espacio, producto de los acontecimientos históricos y los condicionamientos que, desde lo político y lo económico, ha tenido la producción de conocimiento en nuestro país y gran parte del continente latinoamericano. La separación de ambos procesos fue producto de la interrupción el orden constitucional, y la intervención y persecución a la universidad y sus miembros, como parte de la "lucha" contra el "peligro comunista" decretado por la dictadura del General Onganía.

La creación de una institución con las características del CICE - a posteriori de dicha intervención - fue lo que posibilitó la consolidación de ese nuevo perfil profesional del intelectual o especialista en educación, ahora experto. De esta manera los espacios extra-universitarios destinados a la producción de conocimiento, tal es el caso del Instituto Torcuato Di Tella, se convirtieron en un refugio local para el trabajo y desarrollo de equipos que ahora se volcarán a la investigación. Se hace referencia al "refugio local" porque para muchos otros tal refugio fue el exilio.

Así, es posible observar una delimitación "no autónoma" del perfil de experto. Las demandas de producción de conocimiento desde diversos organismos e instituciones, así como la dependencia financiera de centros como el CICE fue una limitante al libre desarrollo del campo. Es por ello que la autonomía -relativa- del mismo no puede garantizarse hasta la recuperación de la estabilidad institucional al interior de las universidades, con la recuperación de la democracia en 1983 y aún luego de ella el proceso tuvo altos y bajos. Junto con esta estabilidad el perfil del experto será redefinido a lo largo de la historia desde nuevos espacios de formación e inserción, así como nuevos condicionamientos y demandas.

Más allá de estas cuestiones, se puede afirmar que la institucionalización de las ciencias de la educación y, posteriormente, la definición del experto/investigador al interior del CICE evidencian claramente los sustentos teóricos, las prácticas y los condicionantes a los que quedó sometido el campo disciplinar y, al mismo tiempo, dejan al descubierto cuáles fueron las condiciones de posibilidad para la consolidación de este en el contexto democrático.

Sin dudas, el CICE se consolidó como un ámbito académico local fundamental para el desarrollo de prácticas de investigación -teórica y empírica- de formación de recursos, de generación de relaciones con diversos tipos de instituciones y también de un perfil técnico, especialista, consultor. En definitiva, como plataforma de despegue de un campo complejo, amplio, en continuo crecimiento y diversificación.

\section{Bibliografía}

ALTAMIRANO, C. Entre el naturalismo y la psicología. el comienzo de la Ciencias Social en la Argentina. In: NEIBURG, F.; PLOTKIN, M. (Comp.). Intelectuales y expertos. La constitución del conocimiento social en la Argentina. Buenos Aires: Paidós, 2004. p. 31-57.

ALTAMIRANO, C.; SARLO, B. Literatura/Sociedad. Buenos Aires: Edicial, 1993. 
BLANCO, A. Razón y modernidad. Gino Germani y la Sociología en la Argentina. Buenos Aires: Siglo XXI editores, 2006.

BUCHBINDER, P. Historia de la Facultad de Filosofía y Letras. Buenos Aires: Editorial Universitaria de Buenos Aires, 1997.

CAMOU, A. Los consejeros del príncipe. Saber técnico y política en los procesos de reforma económica de América Latina. Revista Nueva Sociedad, Buenos Aires, n. 152, p. 54-67, nov./dic. 1997.

CARLINO, F. Transformaciones en la carrera de Ciencias de la Educación de la UBA. Revista del IICE, Buenos Aires, v. 2, n. 3, p. 48-58, dic. 1993.

DUSSEL, I. Víctor Mercante (1870-1934). Perspectivas: revista trimestral de educación comparada, v. 23, n. 3-4, p. 808-821, 1993. DOI: 10.1007/bf02195512.

FREDERIC, S.; GRACIANO, O.; SOPRANO, G. (Coord.). E1 Estado argentino y las profesiones liberales, académicas y armadas. Rosario: Prohistoria, 2010.

GALARZA, D. Los organismos estatales de gobierno y planificación. In: PALAMIDESSI, M.; SUASNABAR, C.; GALARZA, D. (Comps.). Educación, conocimiento y política: Argentina, 1983-2003. Buenos Aires: FLACSO - Manantial, 2007. p. 81-98.

GONZALEZ LEANDRI, R. Las profesiones: entre la vocación y el interés corporativo. Fundamentos para su estudio histórico. Madrid: Catriel, 1999.

HOFSTETTER, R.; SCHNEUWLY, B. Institutionalisation of Educational Sciences and the dynamics of their development. European Educational Research Journal, v. 1, n. 1, p. 3-26, ene./mayo 2002. DOI: 10.2304/eerj.2002.1.1.9.

KING, J. El Di tella y el desarrollo cultural argentino en la década del '60. Buenos Aires: Ediciones de Arte Gaglione, 1985.

KUMMER, V.; BAFFICO, F.; LOPEZ, M.; UGALDE, M. La reapertura de la Facultad de Ciencias de la Educación: 1951-1960. In: OSSANA, E. et al. Historia de la Facultad de Ciencias de la Educación de Paraná 1920-1973. Facultad de Ciencias de la Educación. Paraná: Universidad Nacional de Entre Ríos, 2010.

MENDEZ, J. La Formación de docentes de nivel medio: entre la demanda y la anticipación. Revista Espacios en Blanco, Tandil, n. 21, p. 259-291, 2011.

MENDEZ, J. De intelectuales a expertos. Las ciencias de la educación en la renovación de las ciencias sociales. El caso del centro de investigaciones en ciencias de la educación. Argentina, décadas del '60 y el '70. 2014. 149 f. Disertación (Máster) - Universidad Nacional de Quilmes, Quilmes, 2014. (mimeo).

NEIBURG, F.; PLOTKIN, M. Elites intelectuales y ciencias sociales en la Argentina de los años 60. El Instituto Torcuato Di Tella y la Nueva Economía. Estudios Interdisciplinarios de América Latina y el Caribe, v. 14, n. 1, ene./jun. 2003. Instituto Sverdlin de Historia y Cultura de América Latina, Escuela de Historia Universidad de Tel Aviv. Ramat Aviv, Israel. 
De intelectuales a expertos" en el campo educativo argentino de la década del sesenta...

NEIBURG, F.; PLOTKIN, M. Intelectuales y expertos. La constitución del conocimiento social en la Argentina. Buenos Aires: Paidós, 2004.

OSSANA, E. (Dir.). Historia de la Facultad de Ciencias de la Educación de Paraná 19201973. Facultad de Ciencias de la Educación. Paraná: Universidad Nacional de Entre Ríos, 2010.

PALAMIDESSI, M.; SUASNÁBAR, C.; GALARZA, D. (Comps.). Educación, conocimiento y política: Argentina 1983-2003. Buenos Aires: FLACSO - Manantial, 2007.

SIGAL, S. Intelectuales y poder en la década del sesenta. Buenos Aires: Puntosur, 1991.

SIRVENT, M. T.; LLOMOVATE, S. A cincuenta años de la creación de Ciencias de la Educación. Revista Espacios de crítica y producción, Buenos Aires, n. 36, p. 113-119, nov. 2007.

SOUTHWELL, M. Psicología experimental y Ciencias de la Educación. Notas de historias y fundaciones. La Plata: Edulp, 2003.

SOUTHWELL, M. Algunas características de la formación docente en la historia educativa reciente. El legado del espiritualismo y el tecnocratismo (1955-1976). In: PUIGGROS, A. (Dir.). Dictaduras y utopías en la historia reciente de la educación argentina (1955-1983). Buenos Aires: Galerna, 2003b. p. 105-156.

SUASNABAR, C. Universidad e intelectuales. Educación y política en la Argentina (19551976). Buenos Aires: Manantial, 2004.

SUASNABAR, C. El aporte de la Maestría en Educación de FLACSO en la recomposición del campo intelectual de la educación en la Argentina a comienzos de los años ochenta. Propuesta Educativa, Buenos Aires, v. 2, ano 21, n. 38, p. 39-43, nov. 2012.

SUASNABAR, C. La institucionalización de la educación como campo disciplinar: un análisis desde la perspectiva de la historia social de las ciencias sociales. Revista Mexicana de Investigación Educativa, v. 18, n. 59, p. 1281-1304, oct./dic. 2013.

SUASNABAR, C.; PALAMIDESSI, M. El campo de producción de conocimientos en educación en la Argentina. Notas para una história de la investigación educativa. Revista Educación y Pedagogía, Medellín, v. 18, n. 46, p. 59-77, 2006.

TIRAMONTI, G. Veinte años de democracia: acepciones y perspectivas para la democratización del sistema educativo. In: NOVARO, M.; PALERMO, V. Historia reciente. Argentina en democracia. Buenos Aires: Edhasa, 2004. p. 223-238.

TIRAMONTI, G. Investigación y política educativa en Argentina: dos términos de una ecuación de debilidad institucional. Revista de Estudios Teóricos y Epistemológicos en Política Educativa, v. 1, n. 2, p. 398-414, jul./dic. 2016.

ZIMMERMANN, E. Los liberales reformistas. La cuestión social en la Argentina 18901916. Buenos Aires: Editorial Sudamericana/Universidad de San Andrés, 1995. 
ZIMMERMANN, E.; PLOTKIN, M. Los Saberes de Estado. (Vol. I). Buenos Aires: Ensayo Edhasa, 2012.

ZIMMERMANN, E.; PLOTKIN, M. Las prácticas del Estado. Políticas, sociedad y elites (Vol. II). Buenos Aires: Ensayo Edhasa, 2012.

\section{DOCUMENTOS}

CICE. Memoria y Balance 1966-1967. Publicaciones del ITDT, Buenos Aires, 1967.

CICE. Memoria y Balance 1967-1968, Publicaciones del ITDT, Buenos Aires, 1968

LEYES NACIONALES. Ley de Creación del Consejo Nacional de Investigaciones

Científicas y Técnicas No 1291/58. Anales de Legislación Argentina, Buenos Aires, 1958.

ITDT. Memoria 1960-1962. Publicaciones del ITDT, Buenos Aires, 1962.

Recebido em 13/05/2017

Versão corrigida recebida em 13/06/2017

Aceito em 29/09/2017

Publicado online em 27/07/2017 